\title{
White matter integrity and late-life depression in community-dwelling individuals: diffusion tensor imaging study using tract-based spatial statistics
}

Simone Reppermund, Lin Zhuang, Wei Wen, Melissa J. Slavin, Julian N. Trollor, Henry Brodaty and Perminder S. Sachdev

\section{Background}

Late-life depression has been associated with white matter changes in studies using the regions of interest approach.
Aims
To investigate the cross-sectional and longitudinal relationship between white matter integrity and depression in community-dwelling individuals using diffusion tensor imaging with tract-based spatial statistics.

\section{Method}
The sample comprised 381 participants aged between 72 and 92 years who were assessed twice within 2 years. Depressive symptoms were measured with the Geriatric Depression Scale. Tract-based spatial statistics were applied to investigate white matter integrity in currently depressed $v$. non-depressed elderly people and in those with a history of depression $v$. no history of depression. The relationship between white matter integrity and development of depressive symptoms after 2 years were analysed with logistic regression.

\section{Results}

Individuals with current depression had widespread white matter integrity reduction compared with non-depressed elderly people. Significant fractional anisotropy reductions were found in 45 brain areas with the most notable findings in the frontal lobe, association and projection fibres. A history of depression was not associated with reduced fractional anisotropy. White matter changes in the superior frontal gyrus, posterior thalamic radiation, superior longitudinal fasciculus and in the body of corpus callosum predicted depression at follow-up.

\section{Conclusions}

Reduced white matter integrity is associated with late-life depression and predicts future depressive symptoms whereas a history of depression is not related to white matter changes. Disruption to white matter integrity may be a biomarker to predict late-life depression.

\section{Declaration of interest}

None.
Depression is common in old age and is associated with cognitive impairment, reduced quality of life and increased disability. ${ }^{1,2}$ Neuroimaging studies have suggested white matter hyperintensities, in particular in subcortical prefrontal and temporal brain areas, as one of the primary neurobiological substrates of geriatric depression. ${ }^{3-5}$ A number of studies have shown that white matter changes predict depressive symptoms in older, community-dwelling adults. $^{6-8}$ The authors explain their findings on the basis of the vascular hypothesis of depression in elderly people. According to this hypothesis, cerebrovascular pathologies cause depressive symptoms through disruption of frontosubcortical connections. ${ }^{9}$ However, other studies have not confirmed an association between white matter hyperintensities and the development of depressive symptoms. ${ }^{10}$

Diffusion tensor imaging (DTI) may offer information about the structural integrity of white matter areas and their longitudinal relationship to depressive symptoms. DTI is a relatively new, sensitive and reliable technique for examining white matter microstructure in vivo, thus providing information that can estimate the integrity of pathways within relevant neural networks. A commonly used metric in DTI studies is fractional anisotropy, which estimates the degree to which tissue organisation limits diffusion of water molecules in white matter fibre tracts. ${ }^{11}$ Older patients with major depression demonstrate lower fractional anisotropy values mainly in frontal ${ }^{12,13}$ and temporal brain regions. ${ }^{13}$ Tract-based spatial statistics (TBSS) are designed for examining fibre tracts of the whole brain. This method has superior objectivity and interpretability of DTI analyses than the regions of interest (ROI) method. ${ }^{14}$ The TBSS method analyses the entire brain for group differences or correlations, so it is not restricted to specific brain areas.

Colloby et $a l^{15}$ investigated white matter microstructural changes with TBSS in a sample of patients with late-life depression. They found lower fractional anisotropy in frontal, temporal and midbrain regions compared with healthy controls. However, after correction for multiple comparisons, these differences were no longer statistically significant. Although Bezerra et $a l^{16}$ did not discover significant differences between currently depressed and non-depressed elderly patients using TBSS, two other studies report loss of white matter integrity in the cingulate area and in widespread regions of the frontal and temporal lobes. ${ }^{17,18}$ Two recent meta-analyses ${ }^{19,20}$ and a systematic review on DTI in depression $^{21}$ identified reduced fractional anisotropy in seven brain areas: superior frontal gyrus (left and right), genu of corpus callosum (right), inferior fronto-occipital fasciculus (right), posterior thalamic radiation (right), body of corpus callosum (left), inferior longitudinal fasciculus (right) and superior longitudinal fasciculus (left and right). We report the first prospective study assessing associations between DTI parameters using the TBSS method and depressive symptoms over a 2-year period in a large community sample of older adults. The aims of the present study were to assess whether community-dwelling elderly participants with depressive symptoms demonstrate white matter microstructure abnormalities and whether a lifetime history of depression is associated with white matter microstructure abnormalities. A further aim was to assess whether white matter microstructure abnormalities predict depressive symptoms in a 2-year follow-up period. We expected reduced fractional 
anisotropy in individuals with depressive symptoms and with a history of depression compared with healthy controls. We further hypothesised that a higher degree of white matter microstructure abnormalities predicts the incidence of depression at follow-up.

\section{Method}

\section{Sample}

Participants were recruited from the electoral roll of the Eastern suburbs of Sydney, Australia between 2005 and 2007 as part of an ongoing longitudinal study to examine the prevalence, longitudinal course and risk and protective factors of cognitive impairment and decline in older, community-dwelling individuals (Sydney Memory and Ageing Study, MAS). Detailed methods and recruitment process are published elsewhere. ${ }^{22}$ Briefly, 1037 participants aged between 72 and 92 years were assessed using a detailed neuropsychological and medical assessment. Exclusion criteria were dementia (according to DSM-IV criteria ${ }^{23}$ ), developmental disabilities, psychotic symptoms, schizophrenia or bipolar disorder, multiple sclerosis, motor neuron disease, progressive malignancy and inadequate English to complete a psychometric assessment. The cohort is followed up every 2 years with comprehensive face-to-face assessments. The current study reports data from the first and second follow-up ( 2 and 4 years after baseline, respectively). All participants without contraindications (pacemaker, metallic implant or foreign bodies, cochlear implants, ferromagnetic homeostatic clips, claustrophobia) were invited to undergo magnetic resonance imaging (MRI) scans. A total of 414 participants agreed to and were eligible for MRI brain scans. Participants who did not agree to or were ineligible for an MRI were slightly older than participants who had an MRI scan done $(81.1$ years $($ s.d. $=4.7)$ v. 79.8 years $($ s.d. $=4.6), t=4.4, P<0.001)$. There were no differences between these groups with regard to gender, education or depressive symptoms (all $P>0.05$ ). Of the 414 participants with an MRI scan, 14 had missing Geriatric Depression Scale - 15 item (GDS) ${ }^{24}$ data and 19 participants had too many missing data to classify them as having mild cognitive impairment (MCI), resulting in a total sample of 381 participants. Consensus diagnoses of MCI were made by a panel of psychogeriatricians, neuropsychiatrists, clinical and research neuropsychologists using current international consensus criteria ${ }^{25}$ and 167 participants were classified as having MCI. At the 2-year follow-up, GDS data were available for 353 participants. The study protocol was approved by the ethics committees of the University of New South Wales and the South Eastern Sydney and Illawarra Area Health Service and written informed consent was obtained from each participant.

\section{MRI data acquisition}

All participants underwent MRI scanning on a Philips 3T Achieva Quasar Dual MRI scanner (Philips Medical System, Best, The Netherlands). For each participant, a 32-directional DTI (b1 $=1000$, one b0) scan was acquired with a single-shot, spinecho, echo-planar imaging sequence: echo time $=68 \mathrm{~ms}$, repetition time $=7767 \mathrm{~ms}$, flip angle $=90^{\circ}$, matrix size $=240 \times 240$, field of view $=240 \times 240 \mathrm{~mm}$, yielding in-plane resolution of $1 \times 1 \mathrm{~mm}$, $552.5 \mathrm{~mm}$ contiguous axial slices without gap. Each DTI scan was repeated twice to increase the signal-to-noise ratio.

\section{DTI processing}

DTI data were preprocessed and analysed with the FMRIB's Diffusion Toolbox of the FMRIB's Software Library (FSL) version 4.1.7 (http://www.Fmrib.ox.ac.uk/fsl, Oxford Centre for Functional
Magnetic Resonance Imaging of the Brain, Oxford University, UK). First, each participant's raw DTI data were co-registered and merged together using the FMRIB's linear image registration. Correction for head movement and eddy current distortions was performed on the merged DTI data by linearly registering each diffusion-weighted volume to the first non-diffusion b0 image. Two pre-processed DTI data-sets of each participant were averaged to create a single DTI image. A binary brain mask was created to remove the non-brain tissue using the Brain Extraction Tool in FSL. Subsequently, a diffusion tensor model was fitted to each imaging voxel of the preprocessed DTI data to derive a fractional anisotropy map.

Voxel-wise statistical analyses of fractional anisotropy images were performed using tract-based spatial statistics, ${ }^{14}$ version 1.2 within FSL. First, each participant's fractional anisotropy map was non-linearly aligned to a $1 \times 1 \times 1$ fractional anisotropy target image (FMRIB58_FA) in Montreal Neurological Institute standard space. Second, all aligned fractional anisotropy images were averaged to generate a mean fractional anisotropy image that was then thinned to create a mean tract skeleton. The tract skeleton was further thresholded at fractional anisotropy $>0.2$ to minimise the partial volume effect and cross-participant misregistration. Third, each participant's fractional anisotropy data were projected onto the tract skeleton to create a skeletonised fractional anisotropy map.

\section{Depression measures}

Current depressive symptoms were assessed with the 15-item short form of the GDS, ${ }^{24}$ a self-rating questionnaire shown to be reliable and valid for the assessment of depressive symptoms in elderly people. A higher score indicates more symptoms of depression and a cut-off of six has been established to indicate clinically relevant symptoms of depression. ${ }^{26}$ The GDS does not include somatic and sexual items, and has been validated for use in individuals with mild impairment of cognition. ${ }^{24}$ In the MAS we used the GDS with item 9 as described in Brink (here item 12). ${ }^{27}$ As the GDS is a self-rated questionnaire, there were some missing data. Provided that $80 \%$ or more of the questions were answered, scores were prorated (raw score/items completed $x$ total number of items). This was done for 41 participants $(10.8 \%)$ at time $1\left(T_{1}\right)$ and for 36 participants $(10.2 \%)$ at the 2-year follow-up.

A history of depression was defined as one or more selfreported depressive episodes that had required the attention of a general practitioner, psychologist or psychiatrist and/or previous prescription of antidepressants. A total of 39 participants (10.2\%) were taking antidepressants at time $T_{1}$ and all of these individuals were either in the currently depressed group and/or in the history of depression group

\section{Cardiovascular Risk Factor Index}

The Cardiovascular Risk Factor Index (CVR) is a computed variable based on the research of the Framingham Stroke Study. ${ }^{28}$ It is based on a regression model using current smoking status, diabetic status, systolic blood pressure, total cholesterol level, high-density-lipoprotein level and use of antihypertensive medication. A higher score indicates a higher 10-year risk prediction of a cardiovascular event (coronary death, myocardial infarction, coronary insufficiency, angina, ischemic stroke, hemorrhagic stroke, transient ischemic attack, peripheral artery disease and heart failure). The CVR was available for 369 participants (96.9\% of the sample). 


\section{Statistical analysis}

Statistical analyses included independent $t$-tests and chi-squared tests for comparison of demographic and clinical measures using SPSS 20 for Windows. Voxel-wise statistical analyses were performed using a permutation-based inference program called 'randomise' (with 1000 permutations), which is part of FSL. ${ }^{29}$ Group differences in fractional anisotropy between currently depressed and non-depressed elderly people were assessed using ANOVA implemented in the randomise tool, controlling for age, gender, years of education, MCI and CVR score. Correlation analyses between fractional anisotropy and GDS scores in the whole sample and for individuals who developed depressive symptoms at follow-up were performed using linear regression in the randomise program, adjusting for age, gender, years of education, MCI and CVR score.

Multiple logistic regression was used to analyse whether decreased fractional anisotropy in certain brain areas predicted depression at 2-year follow-up, adjusting for age, gender, years of education, MCI and CVR score. Fractional anisotropy values were converted into $z$-scores. Multiple comparison correction controlling for the family-wise error (FWE) rate was applied to the resulting raw statistical images, using the threshold-free cluster enhancement method implemented in the randomise tool. ${ }^{30}$ The significance threshold was set at $P<0.05$ on the FWE-corrected statistical images. The anatomical location of significant skeletal regions was labelled using the Johns Hopkins University white matter atlas. ${ }^{31}$

\section{Results}

Of the total sample, $6.6 \%(n=25)$ were classified as currently depressed at $T_{1}$ based on a GDS score of 6 or above. The currently depressed group and the non-depressed groups did not differ in age, gender, education, rate of MCI, rate of a lifetime history of depression and CVR score (Table 1).

\section{Group differences in white matter microstructure}

Voxel-wise comparison of the brains of these currently depressed and non-depressed groups of older people revealed significant clusters of reduced fractional anisotropy following permutationbased multiple comparison correction and adjustment for age, gender, education, MCI and CVR score. Online Fig. DS1 and online Table DS1 show brain areas with significantly decreased fractional anisotropy values in the currently depressed group relative to the non-depressed control group. Compared with the non-depressed group, the currently depressed group had widespread reduced fractional anisotropy in 45 different regions.
Online Fig. DS2 illustrates correlations between reduced fractional anisotropy and GDS scores for the whole sample. There were no significant fractional anisotropy reductions in older individuals with a lifetime history of depression $(n=82)$ compared with older individuals without a lifetime history of depression ( $n=293$; all $P>0.05)$.

At follow-up, GDS data were available for 353 individuals. There was no significant change in the mean GDS score for the whole sample $(2.18 \quad$ (s.d.=2.25) v. $2.37 \quad$ (s.d.=2.30), $t($ d.f. $=352)=-1.60, P=0.110)$. A total of 26 individuals had clinically significant symptoms of depression (GDS score of 6 or above) at follow-up. The total number included in the statistical analyses was 317, as 12 had missing CVR data and 24 individuals were currently depressed at $T_{1}$. Of the 317 individuals included in the analyses, 13 developed depression at follow-up.

Voxel-wise comparison of the brains of those who developed depression at follow-up (i.e. GDS score $\geqslant 6$ ) v. those who did not develop depression revealed no significant results following permutation-based multiple comparison correction and adjustment for age, gender, education, MCI and CVR score. This was most likely because of the small number of individuals who developed depression at follow-up, increasing the risk of type II errors. We repeated the analysis using the GDS as a continuous variable to assess whether fractional anisotropy reduction at $T_{1}$ was associated with an increase in depressive symptoms at follow-up, corrected for baseline GDS scores. Reduced fractional anisotropy values with the largest clusters in the frontal lobe, projection fibres and commissural fibres were significantly associated with depressive symptoms 2 years later (see online Table DS2).

In a post hoc analysis, we examined whether reduced fractional anisotropy in the brain areas identified in two recent meta-analyses ${ }^{19,20}$ and a systematic review ${ }^{21}$ predicted depression (GDS score $\geqslant 6$ ) at follow-up. We used logistic regression with currently depressed $v$. not depressed at follow-up as the dependent variable and eight brain areas as predictor variables, adjusted for age, gender, years of education, MCI and CVR score. Table 2 shows the results. Reduced fractional anisotropy in the superior frontal gyrus (left and right), posterior thalamic radiation (right), superior longitudinal fasciculus (left) and body of corpus callosum (left) predicted depression at follow-up.

\section{Discussion}

\section{Comparison with findings from other studies}

The results of this study confirm reduced white matter integrity in currently depressed older, community-dwelling individuals compared with non-depressed individuals. This is in line with previous studies in older patients with depression ${ }^{12,17,18,32-35}$

Table 1 Sample characteristics at baseline

\begin{tabular}{|c|c|c|c|c|c|}
\hline & $\begin{array}{l}\text { Currently depressed group } \\
\qquad(n=25)\end{array}$ & $\begin{array}{l}\text { Non-depressed group } \\
\qquad(n=356)\end{array}$ & $t$ & $\chi^{2}$ & $P$ \\
\hline Age, mean (s.d.) & $80.35(4.44)$ & $79.54(4.49)$ & -0.87 & & 0.383 \\
\hline Gender, male:female & $16: 9$ & $168: 188$ & & 2.64 & 0.104 \\
\hline Years of education, mean (s.d.) & $11.38(3.85)$ & $11.93(3.65)$ & 0.72 & & 0.473 \\
\hline Cardiovascular Risk Factor Index, ${ }^{a}$ mean (s.d.) & $3.32(2.27)$ & $3.85(3.26)$ & 0.80 & & 0.426 \\
\hline Mild cognitive impairment, $n$ (\%) & $13(52)$ & $154(43.3)$ & & 0.73 & 0.394 \\
\hline Geriatric Depression Scale, mean (s.d.) & $8.53(3.01)$ & $1.72(1.28)$ & -22.72 & & $<0.001$ \\
\hline History of depression, ${ }^{\text {b }}$ yes:no & $9: 16$ & $73: 277$ & & 3.13 & 0.077 \\
\hline $\begin{array}{l}\text { a. Data available for } 369 \text { participants only. } \\
\text { b. Data available for } 375 \text { participants only. }\end{array}$ & & & & & \\
\hline
\end{tabular}




\begin{tabular}{|c|c|c|c|}
\hline & Odds ratio $(95 \% \mathrm{Cl})$ & Wald & $P^{a}$ \\
\hline Age & $1.03(0.90-1.19)$ & 0.19 & 0.667 \\
\hline Gender & $1.72(0.44-6.72)$ & 0.61 & 0.436 \\
\hline Education & $0.86(0.70-1.05)$ & 2.24 & 0.135 \\
\hline Cardiovascular Risk Factor Index score & $0.90(0.75-1.07)$ & 1.43 & 0.232 \\
\hline Mild cognitive impairment & $0.78(0.21-2.87)$ & 0.14 & 0.705 \\
\hline Superior frontal gyrus $\mathrm{L}$ & $0.35(0.14-0.89)$ & 4.83 & 0.028 \\
\hline Superior frontal gyrus $\mathrm{R}$ & $4.56(1.57-13.21)$ & 7.82 & 0.005 \\
\hline Genu of corpus callosum $\mathrm{R}$ & $0.51(0.19-1.41)$ & 1.66 & 0.198 \\
\hline Body of corpus callosum L & $3.43(1.05-11.19)$ & 4.16 & 0.041 \\
\hline Inferior fronto-occipital fasciculus $\mathrm{R}$ & $0.85(0.37-1.94)$ & 0.15 & 0.849 \\
\hline Posterior thalamic radiation $\mathrm{R}$ & $0.28(0.11-0.72)$ & 6.91 & 0.009 \\
\hline Superior longitudinal fasciculus $\mathrm{L}$ & $4.22(1.33-13.42)$ & 5.96 & 0.015 \\
\hline Superior longitudinal fasciculus $\mathrm{R}$ & $0.40(0.15-1.07)$ & 3.35 & 0.067 \\
\hline
\end{tabular}

and in population-based samples of older individuals. ${ }^{36}$ Most of the aforementioned studies used the ROI approach and four studies used TBSS. ${ }^{15-18}$ In contrast to the widely used ROI approach, which involves manually placing an ROI over a predefined area and is susceptible to user bias, TBSS includes the whole brain and reduces the problem of misalignment. Previous TBSS study samples comprised selected patients with depression, whereas our study is the first to use TBSS in a community sample of older adults with and without depressive symptoms. Findings from previous TBSS studies in older patients with depression are inconclusive. Whereas two did not find significant differences in white matter integrity between currently depressed and non- depressed elderly people, ${ }^{15,16}$ two reported decreased fractional anisotropy in the corpus callosum, cingulate bundle and corticospinal tract. ${ }^{17,18}$ Our findings show more widespread fractional anisotropy reduction in addition to the aforementioned areas. One explanation could be that our sample was significantly older, with an age range of 72-92 years compared with 59 years and above in other samples. ${ }^{15-18}$ However, we adjusted our analyses for age and still found significant fractional anisotropy reduction in many brain regions in the currently depressed group.

A systematic review of DTI studies in affective disorders ${ }^{21}$ and a recent meta-analysis of voxel-based DTI studies in major depression ${ }^{19}$ suggest that depression can be regarded as a disconnection syndrome within frontal and subcortical areas. Price \& Drevets ${ }^{37}$ suggest that the synaptic transmission through the limbic-cortical-striato-pallido-thalamic circuit related to the medial and orbital prefrontal networks is affected in major depression and this can produce the pathological emotional symptoms.

In contrast to current depressive symptoms, a lifetime history of depression was not associated with reduced fractional anisotropy. However, reduced fractional anisotropy in the superior frontal gyrus (left and right), posterior thalamic radiation (right), superior longitudinal fasciculus (left) and body of corpus callosum (left) predicted depression (GDS score $\geqslant 6$ ) at follow-up. In addition, reduced fractional anisotropy mainly in the frontal lobe, projection fibres and commissural fibres were associated with increased depressive symptoms at follow-up. These findings indicate that reduced white matter integrity in certain brain areas may precede depression. Previous studies reported associations between structural brain abnormalities and poor outcomes of late-life depression ${ }^{38}$ and population-based studies showed that white matter changes can predict depressive symptoms after $1-4$ years. $^{6-8}$ Godin $e t$ al $^{8}$ found, in addition, that severity of white matter lesions was associated with lifetime depression and that white matter lesion increase in the frontal lobe was significantly higher over 4-year follow-up in participants with lifetime depression compared with those without lifetime depression. A possible explanation for the different findings is that the number of participants with a history of depression was larger than in our study and the authors used a standardised interview to diagnose lifetime depression. More longitudinal TBSS studies with larger sample sizes including participants with acute depressive symptoms and those who are in remission are needed to confirm the findings.

TBSS did not reveal significant results for the whole brain comparison between individuals who developed depression at follow-up $v$. those who did not after correction for multiple comparisons. A possible reason for the lack of significant associations is the relatively small number of currently depressed individuals at follow-up, increasing the risk of type II errors. Only 13 individuals developed clinically significant symptoms of depression (a GDS score of 6 or above) at the 2-year follow-up. A longer follow-up period might reveal significant associations even after correction for multiple comparisons. However, reduced fractional anisotropy values at $T_{1}$, mainly in the frontal lobe, projection fibres and commissural fibres, were associated with an increase in depressive symptoms at follow-up.

\section{Link with cardiovascular risk factors and other neuropathological mechanisms}

There is evidence for a link between depression and cardiovascular risk factors. ${ }^{8}$ The vascular depression hypothesis links vascular risk factors/vascular disease to late-life depression. ${ }^{39}$ According to this hypothesis, white matter lesions are caused by cerebrovascular disease disrupting fibre tracts within frontostriatal circuits. ${ }^{9}$ There was no significant difference in the cardiovascular risk profile between currently depressed and non-depressed individuals in our sample. A recent systematic review and meta-analysis suggests that vascular risk factors such as smoking or hypertension, without the actual acute vascular disease, might not precede depression. ${ }^{39}$ White matter lesions increase with age even in the absence of major cerebrovascular risk factors. ${ }^{40}$ Our findings indicate widespread white matter changes in older, currently 
depressed individuals after adjusting for cardiovascular risk factors and for age.

Other possible neuropathological mechanisms causing reduced white matter integrity in depression are (a) elevated cytokine levels that have been associated with reduced neurogenesis and (b) dysregulation of the hypothalamic-pituitary-adrenal axis that leads, with an associated increase in glucocorticoid secretion, to cell death in the hippocampus and to an elimination of activitydependent increases in brain-derived neurotrophic factor (see Murphy \& Frodl $^{20}$ for a review). Glucocorticoids can induce microglial modulation in the central nervous system with consequences such as the release of proinflammatory mediators and disruption of brain networks. ${ }^{41}$

\section{Limitations}

One limitation of this study is the relatively small number of individuals with clinically significant depressive symptoms. We assessed depressive symptoms with the self-rated GDS and not with a standardised clinical interview that would have enabled a clinical diagnosis of depression. However, depressive symptoms not fulfilling rigorous diagnostic criteria are highly prevalent in elderly people and their consequences are similar to those of major depression. ${ }^{1,2}$ There is evidence from previous research that white matter pathology is associated not only with clinical depression but also with the endorsement of depressive symptoms in an older population-based cohort of euthymic individuals, ${ }^{36}$ supporting our findings. Another limitation is that history of depression was measured by self-report during a medical history interview with a trained research assistant. Inevitably, all selfreported data are susceptible to bias and are potentially not as reliable as data derived from clinical records. Finally, the prevalence of MCI in our sample was relatively high. The classification of MCI is susceptible to alterations of criteria for cognitive impairment and the difficulty in operationalising MCI is a limitation of all population-based studies.

In conclusion, our findings demonstrate widespread reduction of white matter integrity in elderly individuals with current depression and suggest that fractional anisotropy reductions precede depressive symptoms. Tract-based spatial statistics might be a useful technique to predict depression in elderly people and may help to explain the pathogenesis of late-life depression. Simone Reppermund, PhD, Lin Zhuang, PhD, Centre for Healthy Brain Ageing,
School of Psychiatry, UNSW Medicine, University of New South Wales, Sydney; School of Psychiatry, UNSW Medicine, University of New South Wales, Sydney;
Wei Wen, PhD, Centre for Healthy Brain Ageing, School of Psychiatry, UNSW Medicine, University of New South Wales and Neuropsychiatric Institute, Prince of Wales Hospital, Sydney; Melissa J. Slavin, PhD, Centre for Healthy Brain Ageing and Dementia Collaborative Research Centre, School of Psychiatry, UNSW Medicine, University of New South Wales, Sydney; Julian N. Trollor, MD, Centre for Healthy Brain Ageing and Department of Developmental Disability Neuropsychiatry, School of Psychiatry, UNSW Medicine, University of New South Wales, Sydney; of Psychiatry, UNSW Medicine, University of New South Wales, Sydney;
Henry Brodaty, MD, DSC, Centre for Healthy Brain Ageing and Dementia Collaborative Research Centre, School of Psychiatry, UNSW Medicine, University of New South Wales and Aged Care Psychiatry, Prince of Wales Hospital, Sydney; Perminder S. Sachdev, MD, PhD, Centre for Healthy Brain Ageing and Dementia Collaborative Research Centre, School of Psychiatry, UNSW Medicine, University of New South Wales, Sydney and Neuropsychiatric Institute, Prince of Wales Hospital, Sydney, Australia

Correspondence: Simone Reppermund, Centre for Healthy Brain Ageing, University of New South Wales, Randwick Campus, Building R1f, NSW 2052, Australia. Email: s.reppermund@unsw.edu.au

First received 10 Apr 2013, final revision 20 Feb 2014, accepted 27 Feb 2014

\section{Funding}

This work was supported by a National Health \& Medical Research Council of Australia (NHMRC) Program Grant (ID 350833) and Capacity Building Grant (ID 568940).

\section{Acknowledgements}

The authors thank the many research assistants and administrative assistants who contributed to data gathering. We are grateful to the participants for their enthusiastic support.

\section{References}

1 Reppermund S, Brodaty H, Crawford JD, Kochan NA, Slavin MJ, Trollor JN, et al. The relationship of current depressive symptoms and past depression with cognitive impairment and instrumental activities of daily living in an elderly population: the Sydney Memory and Ageing Study. J Psychiatr Res 2011; 45: 1600-7.

2 Beekman AT, Deeg DJ, Braam AW, Smit JH, Van Tilburg W. Consequences of major and minor depression in later life: a study of disability, well-being and service utilization. Psychol Med 1997; 27: 1397-409.

3 O'Brien J, Desmond P, Ames D, Schweitzer I, Harrigan S, Tress B. A magnetic resonance imaging study of white matter lesions in depression and Alzheimer's disease. Br J Psychiatry 1996; 168: 477-85.

4 Herrmann LL, Le Masurier M, Ebmeier KP. White matter hyperintensities in late life depression: a systematic review. I Neurol Neurosurg Psychiatry 2008; 79: 619-24.

5 de Groot JC, de Leeuw FE, Oudkerk M, Hofman A, Jolles J, Breteler MM. Cerebral white matter lesions and depressive symptoms in elderly adults. Arch Gen Psychiatry 2000; 57: 1071-6.

6 Teodorczuk A, Firbank MJ, Pantoni L, Poggesi A, Erkinjuntti T, Wallin A, et al. Relationship between baseline white-matter changes and development of late-life depressive symptoms: 3-year results from the LADIS study. Psychol Med 2010; 40: 603-10.

7 Teodorczuk A, O’Brien JT, Firbank MJ, Pantoni L, Poggesi A, Erkinjuntti T, et al. White matter changes and late-life depressive symptoms: longitudinal study. Br J Psychiatry 2007; 191: 212-7.

8 Godin O, Dufouil C, Maillard P, Delcroix N, Mazoyer B, Crivello F, et al. White matter lesions as a predictor of depression in the elderly: the 3C-Dijon study. Biol Psychiatry 2008; 63: 663-9.

9 Alexopoulos GS, Meyers BS, Young RC, Campbell S, Silbersweig D, Charlson M. 'Vascular depression' hypothesis. Arch Gen Psychiatry 1997; 54: 915-22.

10 versluis $\mathrm{CE}$, van der Mast RC, van Buchem MA, Bollen EL, Blauw GJ, Eekhof JA, et al. Progression of cerebral white matter lesions is not associated with development of depressive symptoms in elderly subjects at risk of cardiovascular disease: the PROSPER Study. Int J Geriatr Psychiatry 2006; 21: 375-81.

11 Chua TC, Wen W, Slavin MJ, Sachdev PS. Diffusion tensor imaging in mild cognitive impairment and Alzheimer's disease: a review. Curr Opin Neurol 2008; 21: 83-92.

12 Bae JN, MacFall JR, Krishnan KR, Payne ME, Steffens DC, Taylor WD. Dorsolateral prefrontal cortex and anterior cingulate cortex white matter alterations in late-life depression. Biol Psychiatry 2006; 60: 1356-63.

13 Abe O, Yamasue H, Kasai K, Yamada H, Aoki S, Inoue H, et al. Voxel-based analyses of gray/white matter volume and diffusion tensor data in major depression. Psychiatry Res 2010; 181: 64-70.

14 Smith SM, Jenkinson $M$, Johansen-Berg $H$, Rueckert $D$, Nichols TE, Mackay $\mathrm{CE}$, et al. Tract-based spatial statistics: voxelwise analysis of multi-subject diffusion data. Neuroimage 2006; 31: 1487-505.

15 Colloby SJ, Firbank MJ, Thomas AJ, Vasudev A, Parry SW, O’Brien JT. White matter changes in late-life depression: a diffusion tensor imaging study. J Affect Disord 2011; 135: 216-20.

16 Bezerra DM, Pereira FR, Cendes F, Jackowski MP, Nakano EY, Moscoso MA, et al. DTI voxelwise analysis did not differentiate older depressed patients from older subjects without depression. J Psychiatr Res 2012; 46: 1643-9.

17 Alves GS, Karakaya T, Fusser F, Kordulla M, O’Dwyer L, Christl J, et al. Association of microstructural white matter abnormalities with cognitive dysfunction in geriatric patients with major depression. Psychiatry Res 2012; 203: $194-200$

18 Mettenburg JM, Benzinger TL, Shimony JS, Snyder AZ, Sheline YI. Diminished performance on neuropsychological testing in late life depression is correlated with microstructural white matter abnormalities. Neuroimage 2012; 60: 2182-90.

19 Liao $Y$, Huang $X$, Wu Q, Yang C, Kuang W, Du M, et al. Is depression a disconnection syndrome? Meta-analysis of diffusion tensor imaging studies in patients with MDD. J Psychiatry Neurosci 2013; 38: 49-56.

20 Murphy ML, Frodl T. Meta-analysis of diffusion tensor imaging studies shows altered fractional anisotropy occurring in distinct brain areas in association with depression. Biol Mood Anxiety Disord 2011; 1 : 3. 
21 Sexton $C E$, Mackay $C E$, Ebmeier KP. A systematic review of diffusion tensor imaging studies in affective disorders. Biol Psychiatry 2009; 66: 814-23.

22 Sachdev PS, Brodaty H, Reppermund S, Kochan NA, Trollor JN, Draper B, et al. The Sydney Memory and Ageing Study (MAS): methodology and baseline medical and neuropsychiatric characteristics of an elderly epidemiological non-demented cohort of Australians aged 70-90 years. Int Psychogeriatr 2010; 22: 1248-64.

23 American Psychiatric Association. Diagnostic and Statistical Manual of Mental Disorder (4th edn) (DSM-IV). APA, 1994

24 Yesavage JA, Brink TL, Rose TL, Lum O, Huang V, Adey M, et al. Development and validation of a geriatric depression screening scale: a preliminary report. J Psychiatr Res 1982; 17: 37-49.

25 Winblad B, Palmer K, Kivipelto M, Jelic V, Fratiglioni L, Wahlund LO, et al. Mild cognitive impairment-beyond controversies, towards a consensus: report of the International Working Group on Mild Cognitive Impairment. J Intern Med 2004; 256: 240-6.

26 Herrmann $\mathrm{N}$, Mittmann $\mathrm{N}$, Silver IL, Shulman $\mathrm{KI}$, Busto UA, Shear $\mathrm{NH}$, et al. A validation study of The Geriatric Depression Scale short form. Int I Geriat Psychiatry 1996; 11: 457-60.

27 Brink TL. Geriatric depression and hypochondriasis - incidence, interaction, assessment and treatment. Psychother Theor Res 1982; 19: 506-11.

28 D'Agostino RB, Sr., Vasan RS, Pencina MJ, Wolf PA, Cobain M, Massaro JM, et al. General cardiovascular risk profile for use in primary care: the Framingham Heart Study. Circulation 2008; 117: 743-53.

29 Nichols TE, Holmes AP. Nonparametric permutation tests for functional neuroimaging: a primer with examples. Hum Brain Mapp 2002; 15: 1-25.

30 Smith SM, Nichols TE. Threshold-free cluster enhancement: addressing problems of smoothing, threshold dependence and localisation in cluster inference. Neuroimage 2009; 44: 83-98.

31 Wakana S, Jiang $\mathrm{H}$, Nagae-Poetscher LM, van Zijl PC, Mori S. Fiber tractbased atlas of human white matter anatomy. Radiology 2004; 230: 77-87.
32 Nobuhara K, Okugawa G, Sugimoto T, Minami T, Tamagaki C, Takase K, et al. Frontal white matter anisotropy and symptom severity of late-life depression: a magnetic resonance diffusion tensor imaging study. J Neurol Neurosurg Psychiatry 2006; 77: 120-2.

33 Shimony JS, Sheline YI, D'Angelo G, Epstein AA, Benzinger TL, Mintun MA et al. Diffuse microstructural abnormalities of normal-appearing white matter in late life depression: a diffusion tensor imaging study. Biol Psychiatry 2009; 66: $245-52$.

34 Taylor WD, Macfall JR, Boyd B, Payne ME, Sheline YI, Krishnan RR, et al. One-year change in anterior cingulate cortex white matter microstructure: relationship with late-life depression outcomes. Am J Geriatr Psychiatry 2011; 19: 43-52.

35 Yang Q, Huang $X$, Hong N, Yu X. White matter microstructural abnormalities in late-life depression. Int Psychogeriatr 2007; 19: 757-66.

36 Lamar M, Charlton RA, Morris RG, Markus HS. The impact of subcortical white matter disease on mood in euthymic older adults: a diffusion tensor imaging study. Am J Geriatr Psychiatry 2010; 18: 634-42.

37 Price JL, Drevets WC. Neurocircuitry of mood disorders. Neuropsychopharmacology 2010; 35: 192-216.

38 Alexopoulos GS, Murphy CF, Gunning-Dixon FM, Latoussakis V, Kanellopoulos D, Klimstra S, et al. Microstructural white matter abnormalities and remission of geriatric depression. Am J Psychiatry 2008; 165: 238-44.

39 Valkanova V, Ebmeier KP. Vascular risk factors and depression in later life: a systematic review and meta-analysis. Biol Psychiatry 2013; 73: 406-13.

40 Chowdhury MH, Nagai A, Bokura H, Nakamura E, Kobayashi S, Yamaguchi S. Age-related changes in white matter lesions, hippocampal atrophy, and cerebral microbleeds in healthy subjects without major cerebrovascular risk factors. J Stroke Cerebrovasc Dis 2011; 20: 302-9.

41 Kato TA, Hayakawa K, Monji A, Kanba S. Missing and possible link between neuroendocrine factors, neuropsychiatric disorders, and microglia. Front Integr Neurosci 2013; 7: 53 